\title{
The Pediatric Infectious Disease Journal
}

\section{Antibody persistence in Australian adolescents following meningococcal $\mathrm{C}$ conjugate vaccination \\ --Manuscript Draft--}

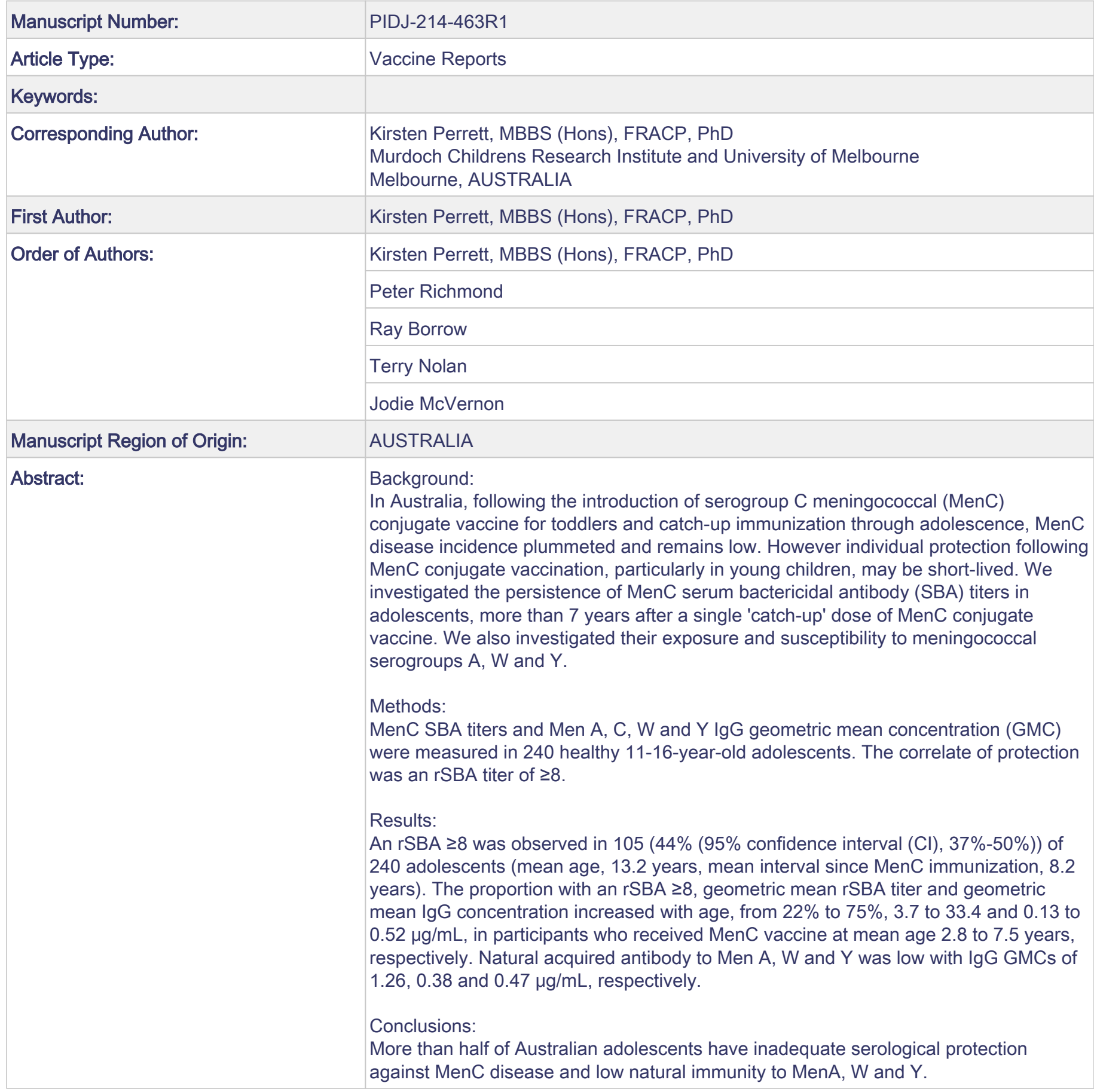


LWW Copyright Transfer and Disclosure Form
Click here to download LWW Copyright Transfer and Disclosure Form: KP_copyrightTransfer.pdf

LWW Copyright Transfer and Disclosure Form
Click here to download LWW Copyright Transfer and Disclosure Form: KP_copyrightTransfer.pdf 


\title{
Antibody persistence in Australian adolescents following meningococcal C
}

\section{conjugate vaccination}

Kirsten P Perrett (MBBS, FRACP, PhD) ${ }^{1}$, Peter C Richmond (MBBS, MRCP, FRACP) ${ }^{2}$, Ray Borrow (PhD, FRCPath) $)^{3}$, Terry Nolan (BMedSc, MBBS, PhD, FRACP, FAFPHM) ${ }^{1}$, Jodie McVernon (BMedSc, MBBS, PhD, FAFPHM) ${ }^{1}$

\begin{abstract}
Affiliations: ${ }^{1}$ Vaccine and Immunisation Research Group (VIRGo), Murdoch
Childrens Research Institute and Melbourne School of Population and Global Health,

The University of Melbourne, Melbourne, Australia, ${ }^{2}$ School of Paediatrics and Child Health, University of Western Australia, Telethon Institute for Child Health Research, Princess Margaret Hospital for Children, Perth, Australia; ${ }^{3}$ Vaccine Evaluation Unit, Public Health England, Manchester Royal Infirmary, Manchester, United Kingdom
\end{abstract}

Address correspondence to: Assoc. Prof. Jodie McVernon

Modelling \& Simulation Group, Centre for Epidemiology \& Biostatistics, Melbourne School of Population and Global Health, Level 5, 207 Bouverie Street, The University of Melbourne 3010, Victoria, Australia. +61 38344 0633, j.mcvernon@unimelb.edu.au

Abbreviated and running head title: MenC antibody in Australian adolescents

\begin{abstract}
Abbreviations: GMC- geometric mean concentration, GMT- geometric mean titer, MenC- serogroup C meningococcal, SBA- serum bactericidal antibody Keywords: Neisseria meningitidis, monovalent serogroup C meningococcal vaccine, persistence of immunity, immunization, adolescence
\end{abstract}


Funding sources: This study was funded by Novartis. Drs Perrett and McVernon are recipients of Research Fellowships from the Australian National Health and Medical Research Council.

Financial disclosures: Dr Perrett has received travel support from Novartis for conference attendance and presentation of data at international meetings and honoraria from Pfizer for educational lectures. Dr Richmond has received institutional funding for investigator-initiated research from GlaxoSmithKline Biologicals and Merck and received travel support from Pfizer and Baxter to present study data at international meetings. Dr Borrow has performed contract research on behalf of Public Health England for Baxter, GSK, Merck, Novartis, Pfizer, Sanofi Pasteur and Sanofi Pasteur MSD. Drs Perrett, Nolan and McVernon's institution (MCRI) has received research grants from GSK, Novartis, CSL, Pfizer and Sanofi Pasteur. Dr Nolan has received past payment for a role (now completed) as a member of the independent data and safety monitoring board for GSK's HPV vaccine. Dr McVernon has been an investigator on vaccine and epidemiological studies sponsored by a range of vaccine manufacturers, and in this role has received support for conference attendance, presentation of data and membership of vaccine advisory boards.

Potential conflict of interest: Dr Richmond has been a member of vaccine advisory boards for Wyeth and CSL Ltd. Dr Nolan chairs the Australian Government's Technical Advisory Group on Immunization (ATAGI) and is a member of the World Health Organization Strategic Advisory Group of Experts (SAGE) on Immunization. Dr McVernon is currently a member of ATAGI. The other authors have no conflicts of interest to disclose. 


\begin{abstract}
Background:

In Australia, following the introduction of serogroup C meningococcal (MenC) conjugate vaccine for toddlers and catch-up immunization through adolescence, MenC disease incidence plummeted and remains low. However individual protection following MenC conjugate vaccination, particularly in young children, may be shortlived. We investigated the persistence of MenC serum bactericidal antibody (SBA) titers in adolescents, more than 7 years after a single 'catch-up' dose of MenC conjugate vaccine. We also investigated their exposure and susceptibility to meningococcal serogroups $\mathrm{A}, \mathrm{W}$ and $\mathrm{Y}$.
\end{abstract}

\title{
Methods:
}

MenC SBA titers and Men A, C, W and Y IgG geometric mean concentration (GMC) were measured in 240 healthy 11-16-year-old adolescents. The correlate of protection was an rSBA titer of $\geq 8$.

\section{Results:}

An rSBA $\geq 8$ was observed in 105 (44\% (95\% confidence interval (CI), 37\%-50\%)) of 240 adolescents (mean age, 13.2 years, mean interval since MenC immunization, 8.2 years). The proportion with an $\mathrm{rSBA} \geq 8$, geometric mean rSBA titer and geometric mean $\operatorname{IgG}$ concentration increased with age, from $22 \%$ to $75 \%, 3.7$ to 33.4 and 0.13 to $0.52 \mu \mathrm{g} / \mathrm{mL}$, in participants who received MenC vaccine at mean age 2.8 to 7.5 years, respectively. Natural acquired antibody to Men A, W and Y was low with IgG GMCs of $1.26,0.38$ and $0.47 \mu \mathrm{g} / \mathrm{mL}$, respectively. 


\section{Conclusions:}

More than half of Australian adolescents have inadequate serological protection against MenC disease and low natural immunity to MenA, W and Y. 


\section{Introduction}

Neisseria meningitidis is a leading cause of meningitis and septicemia globally, with six serogroups responsible for most human disease: A, B, C, W, X and $\mathrm{Y}^{1}$. Over the last century, Australia has experienced both large meningococcal outbreaks and endemic disease. Significant serogroup A meningococcal (MenA) outbreaks occurred in the context of the first and second World Wars, while endemic serogroup B (MenB) and $\mathrm{C}(\mathrm{MenC})$ disease, peaking in the winter months, has prevailed since the $1950 \mathrm{~s}^{2}$. In the late 1990's, almost in parallel to the United Kingdom (UK), Australia observed an increase in the proportion of cases due to MenC among infants, teenagers and young adults. Accordingly, in 2003 monovalent MenC conjugate vaccine was included into the national immunization program ${ }^{3}$ as a single dose at 12 months of age, and a catch-up campaign for all children up to 19 years of age. Program success was unequivocally demonstrated with a decline in MenC disease across all ages (including the unimmunized, indicating herd protection) with no corresponding increase in cases due to other serogroups ${ }^{4}$.

As the UK was the first country to introduce routine MenC vaccination, much has been learned from their experience. Early direct effectiveness estimates of the initial 3-dose infant schedule were promising (over 90\%) ${ }^{5}$. However, cases of clinical vaccine failure indicated that individual protection with an infant only schedule was short-lived ${ }^{6}$, Even after the schedule was amended in 2006 to a 2-dose infant priming and second year of life booster (prime-boost schedule) no evidence of improved antibody persistence or superior protection was demonstrated ${ }^{7,8}$. These observations raised concerns that protection offered by infant and/or early childhood immunization 
may not be sufficient to prevent invasive meningococcal disease through the vulnerable period of adolescence and early adulthood ${ }^{9}$

Surveillance of the phenotype and genotype of cases of meningococcal disease occurs routinely in most developed countries. In both the UK and Australia, there has recently been a modest increase in incidence of serogroup Y meningococcal (MenY) cases. Measurement of natural immunity to other vaccine preventable serogroups (A, $\mathrm{W}$ and $\mathrm{Y}$ ) is vital both to inform appraisal of likely vulnerability to imported strains and to inform national meningococcal vaccine policy.

We measured serum bactericidal antibody (SBA) titers against MenC in 240 adolescents aged 11 to 16 years of age who had received a single catch-up dose of MenC conjugate vaccine in 2003/4 (when aged between 2 and 8 years) to determine the impact on long-term immunity to MenC disease of this program. We also assessed the geometric mean concentration (GMC) of IgG against meningococcal $\mathrm{A}, \mathrm{C}, \mathrm{W}$ and Y to provide information on exposure and susceptibility to these serogroups in Australian children. 


\section{Methods}

\section{Study design and participants}

Between June and December 2011, we collected serum from 240 children in Melbourne and Perth who had received a single catch-up dose of MenC vaccine in 2003/4 (aged between 2 and 8 years). Children were identified through the Australian Childhood Immunization Register (ACIR) as having received one of three licensed MenC conjugate vaccines: MenC-CRM [A] $\left(\right.$ Menjugate $^{\circledR}$, CSL/Novartis Vaccines and Diagnostics); MenC-CRM [B] (Meningitec ${ }^{\circledR}$, Pfizer Australia); or MenC-TT (NeisVac-C ${ }^{\circledR}$, Baxter Healthcare). Each MenC vaccine contains 10 mcg of Neisseria meningitidis group C polysaccharide (strain C11) conjugated to either CRM-197 (a non toxic mutant of diphtheria toxin) (MenC-CRM [A] and MenC-CRM [B]), or tetanus toxoid (MenC-TT). Letters to parents were sent by Medicare Australia.

Potential participants were excluded if they had: received any additional meningococcal vaccines (other than the single catch-up dose of MenC in 2003/4); a known immunodeficiency or chronic illness; received any blood products including immunoglobulin; a previous meningococcal infection; ever received meningococcal chemoprophylaxis; or had antibiotics within the previous seven days.

Participants' individual MenC vaccination history (date and brand) was verified from the parent-held child health record book, ACIR, immunization certificate or General Practitioner records. Written informed consent was obtained from a parent and verbal consent from children aged 12 years and above. Following collection of a $5 \mathrm{~mL}$ blood sample, participants were offered a single dose of quadrivalent serogroup A, C, W and Y meningococcal (MenACWY) conjugate vaccine (Menveo ${ }^{\circledR}$, Novartis 
Vaccines). Menveo is currently licensed in Australia for persons aged 11 years and older. Approval was obtained from ethics committees at the Royal Children's Hospital (30207A) and Princess Margaret Hospital for Children (1877/EP).

\section{Serological responses}

Assays were performed at Public Health England Vaccine Evaluation Unit, Manchester, UK, using standard protocols ${ }^{10}$. Serum was tested for SBA against the O-acetylated MenC strain C11 (C:16:P1.7-1,1) using baby rabbit complement (rSBA). In a subset of $60 \%$ (determined by sampling specimens across the range of titre distributions to assess correlation across a full spectrum of values), assays were repeated using human complement (hSBA). SBA titers were expressed as the final serum dilution in which $50 \%$ bacterial killing was observed after 60 minutes ${ }^{11}$. The lower limit of detection was a titer of 4 . Values below 4 were assigned a value of 2 . Serogroup-specific serum IgG antibody concentrations to serogroup A, C, W and Y meningococci were measured by enzyme linked immunosorbent assays (ELISAs) with lower limits of detection of $0.08,0.06,0.065$ and $0.075 \mu \mathrm{g} / \mathrm{ml}$, respectively.

\section{Statistical analysis}

The primary objective was to determine the proportion who had a MenC rSBA titer of $\geq 8$ (the putative protective threshold) ${ }^{12}$ more than seven years after vaccination. It was hypothesized that rSBA titers against MenC would have waned, leaving $50 \%$ or more of adolescents without demonstrable protection. A sample size of 240 participants would demonstrate this proportion with $95 \%$ confidence intervals (CI) $43.5 \%, 56.5 \%$. Secondary objectives were to calculate the: proportion with a MenC hSBA titer of $\geq 4{ }^{13}$ and $\geq 8$ (in $60 \%$ subset); MenC SBA geometric mean titers 
(GMT); and IgG geometric mean concentrations (GMC) against meningococcal serogroups A, C, W and Y. Analysis was conducted on all available data.

We used univariate and multivariate logistic regression to assess the impact of age at MenC immunization, sex of participant and brand of MenC vaccine on achievement of assay titers above the putative protective thresholds $\mathrm{rSBA} \geq 8^{12}$ and hSBA $\geq 4^{13}$. Further, we performed univariate and multivariate linear regression analysis for log rSBA and hSBA titers and MenC IgG concentrations, to examine the influence of the same variables across the full range of assay results. All analyses were carried out using Microsoft Excel ${ }^{\circledR}$ (version 14.3.9) and STATA (StataCorp ${ }^{\circledR}$, version 11). 


\section{Results}

Of the almost 7000 families approached, 466 responded, 293 were assessed for eligibility and 53 excluded. Reasons for exclusion included: no documentation of MenC vaccine, receipt of more than 1 previous MenC vaccine, long-term antibiotics, outside of age group or too busy). All 240 enrolled participants (160 from Melbourne and 80 from Perth) had blood available for analysis. The mean age at enrolment was 13.2 years (range, 11.1 to 16.8), mean interval since MenC immunization was 8.2 years (standard deviation, \pm 0.54 ) and mean age at receipt of MenC vaccine was 5.1 years (range, 2.4 to 8.8 ) (Table I).

Overall in 2003/4, 189 participants were immunized with MenC-TT, 21 with MenCCRM [A], 21 with MenC-CRM [B] and for 9 participants no brand of MenC vaccine was recorded (Table III). The breakdown by type of MenC vaccine used in each location was 117/20/19/4 for Melbourne and 72/1/2/5 for Perth for MenC-TT, MenCCRM [A], MenC-CRM [B] and Unknown respectively. MenC-TT recipients were significantly older than those who received MenC-CRM [A] (mean age 5.1 years compared to 4.5 years, linear regression coefficient -0.65 (95\% CI $-1.29,0.00), \mathrm{p}=$ 0.049). At the completion of the study, $231(96 \%)$ of 240 participants received MenACWY conjugate vaccine.

\section{Primary objective}

Overall, more than seven years following MenC vaccination, 105 (44\% (CI 37\%$50 \%$ )) of 240 adolescents had an rSBA titer $\geq 8$ (range, $22 \%$ to $75 \%$ in participants who received MenC at mean age 2.8 and 7.5 years, respectively (Table II, Figure 1). An rSBA titer $\geq 8$ was found in 44 of $134(33 \%)$ adolescents who received MenC 
vaccine aged 2 to 4 years compared with 61 of $106(58 \%)$ adolescents who received MenC vaccine aged 5 to 8 years.

\section{Secondary objectives}

Overall the MenC rSBA GMT was 11.3 (CI 8.7-14.8) and ranged from 3.7 to 33.4 in participants who had MenC vaccine at mean age 2.8 and 7.5 years, respectively (Table II, Supplementary Figure 1). The MenC rSBA GMT was significantly lower (6.89 (CI 4.96-9.56)) in adolescents who received MenC vaccine at 2 to 4 years of age compared with those who received MenC vaccine at 5 to 8 years of age (21.20 (CI $14.0-32.1)(\mathrm{p}<0.0001)$

A MenC hSBA titer $\geq 4$ was found in 48 of 140 (34\% (CI 26\%-42\%)) and a titer $\geq 8$ in 39 of 140 (28\% (CI 20\%-35\%)) adolescents (data not shown).

Overall, the MenC IgG GMC was $0.29 \mu \mathrm{g} / \mathrm{mL}$ (CI 0.25-0.33). Adolescents who received MenC vaccine at 2 to 4 years of age had a significantly lower MenC IgG GMC $(0.24 \mu \mathrm{g} / \mathrm{mL}(\mathrm{CI} 0.20-0.29))$ than adolescents who received MenC vaccine between 5 and 8 years of age $(0.37 \mu \mathrm{g} / \mathrm{mL}($ CI $0.30-0.45))(\mathrm{p}=0.0009)$.

\footnotetext{
Age at MenC immunization was the only parameter consistently shown to significantly affect the MenC rSBA titer, IgG concentration and proportion with rSBA $\geq 8$, both by univariate and multivariate analysis (adjusting for sex of participant and brand of MenC vaccine) (Table IVa and b). There were no significant differences in these immune measures by participant sex.
} 
There was a trend (not statistically significant) for those who received a tetanustoxoid conjugate MenC vaccine (MenC-TT), rather than a CRM-197 conjugate MenC vaccine (MenC-CRM [A] or MenC-CRM [B]) to exhibit higher rSBA titers (rSBA titre and proportion with an rSBA titer $\geq 8$ ) (Table III). This reached statistical significance for MenC hSBA titres using human complement (hSBA titre and proportion with an hSBA titer $\geq 4$ ) (Table IVb).

Men A, W and Y IgG GMCs in the 11 to 16-year-old participants were 1.26, 0.38 and $0.47 \mu \mathrm{g} / \mathrm{mL}$, respectively and did not differ by age (data not shown). 


\section{Discussion}

\section{Key findings}

This study shows that sustained vaccine-induced immunity to MenC disease during childhood is dependent on age at vaccination. Further, more than half of the current cohort of Australian adolescents may be susceptible to MenC disease. Specifically, only $44 \%$ of the 11 to 16 -year-olds in this study had persistent rSBA titers above the serological correlate of protection (rSBA titer of $\geq 8){ }^{12}$ more than seven years after immunization. Long-term persistence of MenC antibody (rSBA, hSBA and IgG) following early childhood immunization improved with increasing age at primary vaccination (between 2 to 8 years of age). This is also the first study to show that natural immunity to other vaccine-preventable serogroups of meningococci (A, W and Y) is low in 11 to 16-year-old Australian adolescents.

The age-dependent MenC antibody findings of this study concur with previous evidence of poor antibody persistence following MenC vaccination in young children ${ }^{14,15}$ and improved antibody persistence in older children ${ }^{16}$. In our study, only one third of the adolescents immunized between 2 and 4 years of age had an rSBA titer $\geq 8$, compared to almost $60 \%$ of adolescents immunized between 5 to 8 years of age. MenC rSBA titers and IgG concentrations followed a similar pattern providing further evidence that immune responses to meningococcal vaccines improve around the age of 5 years ${ }^{14-17}$ regardless of whether administered as a priming or booster dose ${ }^{7}$.

Human complement source SBA assays were performed in the original studies of SBA against MenC. A serum dilution $\geq 4$ by hSBA is an historical population-level correlate of naturally induced immune protection ${ }^{13}$, whilst a serum dilution of $\geq 8$ by 
rSBA allows bridging comparison with population-derived correlates of direct protective efficacy following conjugate vaccine administration ${ }^{18}$. Only $34 \%$ of adolescents in our study had a MenC hSBA titer $\geq 4$ compared to $44 \%$ with a rSBA titer $\geq 8$. This finding is consistent with previous evaluations of meningococcal correlates of protection that rSBA may give higher titres than hSBA and further, although a rSBA $\leq 8$ predicts susceptibility (with high specificity) and a hSBA $\geq 4$ indicates protection, a hSBA $\leq 4$ does not always indicate susceptibility ${ }^{11,13}$.

\section{Explanation for findings}

Immunological maturation has emerged as the most compelling explanation for agedependent MenC antibody responses in childhood. Primary antibody responses to MenC conjugate vaccination are higher in adolescents than in younger children ${ }^{19}$. Further, natural decline in circulating MenC SBA titers with time has been shown in infants and likely occurs faster in younger children than adolescents ${ }^{20}$. Younger children also appear to be less well primed for a secondary immune response ${ }^{14}$. Immature intrinsic germinal center B-cell reactions and suboptimal microenvironmental factors in lymphoid tissue and bone marrow are thought to be some of the mechanisms behind these observations ${ }^{21}$, culminating in generation of fewer long-lived plasma cells ${ }^{22}$.

\section{Strengths and Weaknesses}

In contrast to data from meningococcal serosurveys conducted using banked residual sera agnostic to vaccine status in the UK and Netherlands ${ }^{8,17,23,24}$, this study selectively recruited individuals with a confirmed date and brand of MenC vaccination. This study therefore allowed accurate determination of antibody 
persistence following MenC immunization in childhood by age and product. The uptake of MenC vaccine in the catch-up group (those aged 2 to 18 years) in Australia between 2003 and 2006 is estimated at $70 \%{ }^{25}$. Therefore, it is possible that our findings may underestimate the true susceptibility to MenC disease in the current Australian adolescent population.

This study was not powered to look at persistence of the immune response following childhood MenC immunization by brand of vaccine received, so interpretation of this analysis is limited due to the small sample size of adolescents primed with either of the MenC-CRM vaccines. Our data suggested a trend for better persistence following receipt of the MenC-TT tetanus toxoid conjugate MenC vaccine than either of the CRM conjugates for rSBA and statistically significantly by hSBA. Other studies have found superior immune responses following primary vaccination with the MenC-TT vaccine than either of the MenC-CRM vaccines ${ }^{7,19}$. Larger cohort studies following recipients of alternative MenC vaccines would be required to make definitive conclusions regarding the influence of conjugate protein type on long-term persistence of MenC antibody in childhood.

Despite the strongly significant age-dependent antibody findings of this study, the MenC rSBA GMT and proportion with rSBA titer $\geq 8$ were lower (non-statistically significant) in the children immunized with MenC at 8 years compared to 7 years of age. This is likely due to the small sample size in the older age-group (resulting in very wide CI), and limits the accuracy of the data for that age. Accordingly, for both age groups with small sample sizes ( 2 and 8 years of age) data was combined with the adjacent age group in figure 1 . 
This study also indicates that the current cohort of Australian adolescents may be susceptible to other meningococcal serogroups. Although no definitive surrogate of protection has been defined for serogroups A, W or Y, IgG concentrations can be informative. MenW and Y IgG antibody concentrations were low and showed little variation by age in the 11 to 16 -year-old adolescents. A recent seroprevalence study of MenW and Y rSBA in England also found natural immunity to be low particularly in young children, with no rise in adolescence. Instead, peak titers to these strains were observed in 20 to 24 -year-olds (W-135) and 30 to 44 -year-olds (Y) ${ }^{24}$. Historically, serogroup Y disease has been rare in the UK and Australia, but recently such cases have more than doubled both in the UK (from 29 in 2007/8 to 81 in $2011 / 12$, an increase from $2 \%$ to $11 \%$ of all meningococcal cases) ${ }^{26,27}$ and in Australia (from 7 in 2008 to 15 in 2011 , a rise from $2.8 \%$ to $7.0 \%$ of all cases) ${ }^{28,29}$. Therefore, the low levels of natural immunity to serogroup Y are of some concern. Further, a recent study in university students in Nottingham, UK, found carriage rates of MenY have increased dramatically from 5\% in $1999-2001^{30}$ to up to $25 \%$ in 2009 31,32 suggesting MenY circulation in the UK may be increasing. No such Australian adolescent carriage studies have been performed. In our study, serogroup A IgG levels were higher than for MenW or Y despite no evidence of circulation in Australia ${ }^{28}$. Similar seroprevalence findings were reported in the $\mathrm{UK}^{23}$. These higher MenA concentrations may be attributable to the presence of cross-reacting antigens ${ }^{33-35}$.

\section{Clinical relevance}

In accordance with predictions, aging cohorts of children immunized with MenC vaccine in infancy and early childhood has been associated with an overall increase in 
the pool of apparently susceptible older children and adolescents ${ }^{14,36}$. Serial UK seroprevalence studies show that the high levels of MenC immunity observed in 10 to 19-year-olds in 2000-2004, representing those eligible for catch-up vaccination during primary and secondary school ${ }^{23}$, persisted as they aged into the 15 to 24 -year-old group by 2009,10 years following the catch-up campaign ${ }^{8}$. In that same year, however, less than $30 \%$ of UK children aged from 1 to 14 years had persistent MenC SBA titers $\geq 8$. These lower levels of seroprotection in the early teenage cohort in 2009 raise concerns that herd immunity, the predominant mechanism of disease control at present, will decline as catch-up cohorts continue to age ${ }^{37}$. Accordingly, over the next 10 years, the likelihood of re-establishment of endemic transmission of imported strains will inevitably increase.

Although we did not measure persistence of immunity in children who had been immunized at the routine Australian MenC vaccination time-point of 12 months, our antibody results were strongly age-dependent. More than seven years following MenC vaccination, only $22 \%$ of our youngest study participants (mean age at receipt of MenC, 2.8 years) had an rSBA titer $\geq 8$, suggesting that the proportion of children with titers considered protective after receipt of MenC vaccine at 12 months would be even lower. Recently, a study of antibody persistence 5 years following either a single dose of combined Haemophilus influenzae type b-MenC or monovalent MenC conjugate vaccines at 12 to 18 months of age, reported only $19 \%$ and $25 \%$ of children respectively, had an rSBA titer $\geq 8$, similar levels to our study ${ }^{38}$. We predict that by 2015, less than 1 in 5 Australian children entering adolescence (12 years of age) will have MenC rSBA titers sufficient for protection against MenC disease. 
Circulation of MenC in Australia is currently low (only 11 laboratory-confirmed cases in 2012) ${ }^{39}$ and comparable to cases of $\mathrm{W}$ and $\mathrm{Y}$ ( 7 and 15 cases in 2012 respectively) ${ }^{39}$. By this measure alone, introduction of an adolescent meningococcal booster (MenC or MenACWY) may not be justified at this time. However in 2012, 3 cases of MenC disease occurred in the immunized cohort (15 to 24 years of age) ${ }^{39}$ and at least 5 cases of vaccine failure have occurred in children since the introduction of MenC vaccine in Australia (personal communication). What is not yet known, but critical for a formal cost-effectiveness evaluation of routine adolescent meningococcal vaccination in Australia, is the rate of carriage of circulating strains in adolescents and young adults (the major transmitters of disease in the pre-vaccine era). If the rate of endemic transmission is increasing in this cohort, as predicted with low levels of seroprotection, herd immunity (a significant component of the effectiveness of MenC vaccines) will decrease. In addition, even with appropriate antibiotics, meningococcal disease progresses rapidly resulting in an often devastating and potentially fatal course. Accordingly, due to the risk of a return to pre-vaccine disease patterns in adolescents, a preemptive adolescent booster meningococcal strategy in Australia should be considered. The dual benefit of this strategy is direct protection to the immunized adolescent going into the secondary peak risk period in the late teen years and early twenties and also indirect protection of these emergent parents to infants. In the UK, with the considerable MenC disease outbreak of the early 2000s still in recent memory, a preemptive adolescent MenC vaccine strategy has commenced ${ }^{40}$. A catchup campaign was implemented for all new starters at universities up to the age of 25 years ${ }^{40}$. In the US, the incidence of meningococcal disease has declined among all age groups and in all vaccine-related serogroups since a single-dose of MenACWY conjugate vaccine was recommended at 11-12 years of age in $2005^{41}$. Recently, the 
Advisory Committee on Immunization Practices (ACIP) revised the recommendation to a 2-dose strategy to optimize meningococcal protection through late adolescence into early adulthood ${ }^{42}$.

Our study has revealed both the current and potential future susceptibility of Australian adolescents to serogroups $\mathrm{A}, \mathrm{C}, \mathrm{W}$ and $\mathrm{Y}$, and provides new evidence to inform considerations of meningococcal vaccine schedule requirements. 


\section{Acknowledgements}

We are grateful to the participants of this study and their family members. We thank the contributions of the staff members of the Vaccine and Immunisation Research Group (Melbourne), Marita Kefford, Sharon Trevorrow, Mairead Phelan, Annmarie McEvoy, Jane Ryrie, Clare Brophy, Janet Briggs, Marie West, Jacinta Sonego, Jacinta O'Keefe, Judith Spotswood, Paula Nathan and Bernie McCudden; Caroline Talbot and Jennifer Kent of the Vaccine Trials Group (Perth); and Drs Helen Findlow and Xilian Bai of the Public Health England Vaccine Evaluation Unit (Manchester, UK). 


\section{References}

1. Stephens DS, Greenwood B, Brandtzaeg P. Epidemic meningitis, meningococcaemia, and Neisseria meningitidis. Lancet. Jun 30 2007;369(9580):2196-2210.

2. Patel MS. Australia's century of meningococcal disease: development and the changing ecology of an accidental pathogen. The Medical journal of Australia. Feb 5 2007;186(3):136-141.

3. Booy R, Jelfs J, El Bashir H, Nissen M. Impact of meningococcal C conjugate vaccine use in Australia. The Medical journal of Australia. 2007;186(3):108-109.

4. Tapsall J. Annual report of the Australian meningococcal surveillance programme, 2007 - amended. Comm Dis Intell. 2009;33(1):1-9.

5. Ramsay ME, Andrews NJ, Trotter CL, Kaczmarski EB, Miller E. Herd immunity from meningococcal serogroup $C$ conjugate vaccination in England: database analysis. $B M J$ (Clinical research ed. Feb 15 2003;326(7385):365-366.

6. Trotter C, Andrews N, Kaczmarski E, Miller E, Ramsay M. Effectiveness of meningococcal serogroup $\mathrm{C}$ conjugate vaccine 4 years after introduction. Lancet. 2004;364:365-367.

7. Borrow R, Andrews N, Findlow H, et al. Kinetics of antibody persistence following administration of a combination meningococcal serogroup $\mathrm{C}$ and haemophilus influenzae type $\mathrm{b}$ conjugate vaccine in healthy infants in the United Kingdom primed with a monovalent meningococcal serogroup $\mathrm{C}$ vaccine. Clin Vaccine Immunol. Jan 2010;17(1):154-159.

8. Ishola DA, Jr., Borrow R, Findlow H, Findlow J, Trotter C, Ramsay ME. Prevalence of serum bactericidal antibody to serogroup $\mathrm{C}$ Neisseria meningitidis in England a decade after vaccine introduction. Clin Vaccine Immunol. Aug 2012;19(8):1126-1130.

9. Trotter C, R B, Findlow J, et al. Seroprevalence of antibodies against serogroup C meningococci in England in the postvaccination era. Clin Vacc Immunol. 2008;15(11):1694-1698.

10. Maslanka SE, Gheesling LL, Libutti DE, et al. Standardization and a multilaboratory comparison of Neisseria meningitidis serogroup A and C serum bactericidal assays. The Multilaboratory Study Group. Clinical and diagnostic laboratory immunology. Mar 1997;4(2):156-167.

11. Borrow R, Andrews N, Goldblatt D, Miller E. Serological basis for use of meningococcal serogroup $\mathrm{C}$ conjugate vaccines in the United Kingdom: reevaluation of correlates of protection. Infection and immunity. Mar 2001;69(3):1568-1573.

12. Andrews N, Borrow R, Miller E. Validation of serological correlate of protection for meningococcal $\mathrm{C}$ conjugate vaccine by using efficacy estimates from postlicensure surveillance in England. Clinical and diagnostic laboratory immunology. Sep 2003;10(5):780-786.

13. Goldschneider I, Gotschlich EC, Artenstein MS. Human immunity to the meningococcus. I. The role of humoral antibodies. The Journal of experimental medicine. Jun 1 1969;129(6):1307-1326.

14. Perrett KP, Winter AP, Kibwana E, et al. Antibody persistence after serogroup C meningococcal conjugate immunization of United Kingdom primary-school children in 1999-2000 and response to a booster: a phase 4 clinical trial. Clin Infect Dis. Jun 15 2010;50(12):1601-1610.

15. Snape MD, Kelly DF, Green B, Moxon ER, Borrow R, Pollard AJ. Lack of serum bactericidal activity in preschool children two years after a single dose of serogroup C 
meningococcal polysaccharide-protein conjugate vaccine. The Pediatric infectious disease journal. Feb 2005;24(2):128-131.

16. Snape MD, Kelly DF, Lewis $\mathrm{S}$, et al. Seroprotection against serogroup $\mathrm{C}$ meningococcal disease in adolescents in the United Kingdom: observational study. BMJ (Clinical research ed. Jun 28 2008;336(7659):1487-1491.

17. de Voer RM, Mollema L, Schepp RM, et al. Immunity against Neisseria meningitidis serogroup $\mathrm{C}$ in the Dutch population before and after introduction of the meningococcal $\mathrm{c}$ conjugate vaccine. PLoS ONE. 2010;5(8):e12144.

18. Arguedas A, Soley C, Loaiza C, et al. Safety and immunogenicity of one dose of MenACWY-CRM, an investigational quadrivalent meningococcal glycoconjugate vaccine, when administered to adolescents concomitantly or sequentially with Tdap and HPV vaccines. Vaccine. Apr 19 2010;28(18):3171-3179.

19. Burrage $M$, Robinson $A$, Borrow $R$, et al. Effect of vaccination with carrier protein on response to meningococcal $\mathrm{C}$ conjugate vaccines and value of different immunoassays as predictors of protection. Infection and immunity. Sep 2002;70(9):4946-4954.

20. Trotter CL, Andrews NJ, Kaczmarski EB, Miller E, Ramsay ME. Effectiveness of meningococcal serogroup C conjugate vaccine 4 years after introduction. Lancet. Jul 2430 2004;364(9431):365-367.

21. Siegrist CA, Aspinall R. B-cell responses to vaccination at the extremes of age. Nature reviews. Mar 2009;9(3):185-194.

22. Perrett KP, Jin C, Clutterbuck E, et al. B cell memory to a serogroup C meningococcal conjugate vaccine in childhood and response to booster: little association with serum IgG antibody. J Immunol. Sep 1 2012;189(5):2673-2681.

23. Trotter CL, Borrow R, Findlow J, et al. Seroprevalence of antibodies against serogroup C meningococci in England in the postvaccination era. Clin Vaccine Immunol. Nov 2008;15(11):1694-1698.

24. Trotter CL, Findlow H, Borrow R. Seroprevalence of serum bactericidal antibodies against group W135 and Y meningococci in England in 2009. Clin Vaccine Immunol. Feb 2012;19(2):219-222.

25. Meningococcal vaccines for Australians. NCIRS Fact Sheet. 2009. http://www.ncirs.edu.au/immunisation/fact-sheets/meningococcal-vaccines-factsheet.pdf.

26. Health Protection Agency U. Invasive meningococcal infections laboratory reports, England and Wales by capsular group \& epidemiological year, 1998/99-2011/12. 2012; http://www.hpa.org.uk/webc/HPAwebFile/HPAweb_C/1317136087064. Accessed 20 June, 2013.

27. Ladhani SN, Flood JS, Ramsay ME, et al. Invasive meningococcal disease in England and Wales: implications for the introduction of new vaccines. Vaccine. May 21 2012;30(24):3710-3716.

28. Lahra MM, Enriquez RP. Annual report of the Australian Meningococcal Surveillance Programme, 2011. Commun Dis Intell. Sep 2012;36(3):E251-262.

29. Tapsall J. Annual report of the Australian Meningococcal Surveillance Programme, 2008. Communicable diseases intelligence quarterly report. Sep 2009;33(3):259-267.

30. Maiden MC, Ibarz-Pavon AB, Urwin R, et al. Impact of meningococcal serogroup C conjugate vaccines on carriage and herd immunity. The Journal of infectious diseases. Mar 1 2008;197(5):737-743.

31. Ala'aldeen DA, Oldfield NJ, Bidmos FA, et al. Carriage of meningococci by university students, United Kingdom. Emerging infectious diseases. Sep 2011;17(9):1762-1763. 
32. Bidmos FA, Neal KR, Oldfield NJ, Turner DP, Ala'Aldeen DA, Bayliss CD. Persistence, replacement, and rapid clonal expansion of meningococcal carriage isolates in a 2008 university student cohort. Journal of clinical microbiology. Feb 2011;49(2):506-512.

33. Guirguis N, Schneerson R, Bax A, et al. Escherichia coli K51 and K93 capsular polysaccharides are crossreactive with the group A capsular polysaccharide of Neisseria meningitidis. Immunochemical, biological, and epidemiological studies. The Journal of experimental medicine. Dec 1 1985;162(6):1837-1851.

34. Myerowitz RL, Gordon RE, Robbins JB. Polysaccharides of the genus Bacillus crossreactive with the capsular polysaccharides of Diplococcus pneumoniae type 3, Haemophilus influenzae type b, and Neisseria meningitidis group A. Infection and immunity. Dec 1973;8(6):896-900.

35. Robbins JB, Myerowitz L, Whisnant JK, et al. Enteric bacteria cross-reactive with Neisseria meningitidis groups $\mathrm{A}$ and $\mathrm{C}$ and Diplococcus pneumoniae types I and 3. Infection and immunity. Nov 1972;6(5):651-656.

36. Pollard AJ, Perrett KP, Beverley PC. Maintaining protection against invasive bacteria with protein-polysaccharide conjugate vaccines. Nature reviews. Mar 2009;9(3):213-220.

37. Campbell H, Andrews N, Borrow R, Trotter C, Miller E. Updated postlicensure surveillance of the meningococcal $\mathrm{C}$ conjugate vaccine in England and Wales: effectiveness, validation of serological correlates of protection, and modeling predictions of the duration of herd immunity. Clin Vaccine Immunol. May 2010;17(5):840-847.

38. Booy R, T. Nolan, G. Reynolds, P. Richmond, M. Nissen, H. Marshall, T. Stoney, M. Van Der Wielen, D. Kolhe, J. Miller. Immunogenicity and safety of the Hib-MenC-TT conjugate vaccine in Hib-primed toddlers: 5 year follow-up. 8th World Congress of the World's Society for Pediatric Infectious Diseases (WSPID). Vol 0635. Cape Town, South Africa2013.

39. Lahra MM, Enriquez RP. Australian Meningococcal Surveillance Programme annual report, 2012. Communicable diseases intelligence quarterly report. 2013;37(3):E224232.

40. England PH. Changes to the Meningococcal C conjugate (MenC) vaccine schedule 2013. 2013; https://http://www.gov.uk/government/uploads/system/uploads/attachment_data/file/1942 18/PHE_MenC_QAs_acc.pdf. Accessed June 28, 2013.

41. Bilukha OO, Rosenstein N. Prevention and control of meningococcal disease. Recommendations of the Advisory Committee on Immunization Practices (ACIP). MMWR Recomm Rep. May 27 2005;54(RR-7):1-21.

42. Cohn AC, MacNeil JR, Clark TA, et al. Prevention and control of meningococcal disease: recommendations of the Advisory Committee on Immunization Practices (ACIP). MMWR Recomm Rep. Mar 22 2013;62(RR-2):1-28. 


\section{Figure Legends}

Figure 1: Proportion with $r S B A \geq 8(95 \% \mathrm{CI})$, more than 7 years following MenC vaccine, shown by age at primary MenC vaccination

Supplementary Figure 1: MenC SBA geometric mean titers (95\% CIs) 7 to 8 years following MenC vaccine, shown by age at primary MenC vaccination 
Table I: Demographic characteristics of enrolled participants, by age at primary serogroup C meningococcal (MenC) vaccine

\begin{tabular}{|c|c|c|c|c|c|c|c|c|}
\hline Age at MenC vaccine & 2 years & 3 years & 4 years & 5 years & 6 years & 7 years & 8 years & $\begin{array}{c}\text { All } \\
2 \text { to } 8 \text { years }\end{array}$ \\
\hline $\mathbf{N}$ & 9 & 58 & 67 & 33 & 50 & 16 & 7 & 240 \\
\hline \multicolumn{9}{|l|}{ Age at enrolment, years } \\
\hline Mean \pm SD & $11.43 \pm 0.21$ & $12.02 \pm 0.44$ & $12.85 \pm 0.48$ & $13.35 \pm 0.66$ & $14.31 \pm 0.60$ & $15.55 \pm 0.57$ & $15.97 \pm 0.23$ & $13.24 \pm 1.27$ \\
\hline Range & $(11.05-11.83)$ & $(11.21-13.34)$ & $(11.67-13.82)$ & $(11.88-14.54)$ & $(12.84-15.61)$ & $(14.55-16.78)$ & $(15.62-16.31)$ & $(11.05-16.78)$ \\
\hline \multicolumn{9}{|l|}{ Sex No. (\%) } \\
\hline Male & $4(44.4)$ & $32(55.2)$ & $39(58.2)$ & $20(60.6)$ & $32(64.0)$ & $10(62.5)$ & $4(57.1)$ & $141(58.8)$ \\
\hline \multicolumn{9}{|c|}{ Interval since MenC vaccine } \\
\hline Mean \pm SD & $8.64 \pm 0.33$ & $8.46 \pm 0.34$ & $8.29 \pm 0.44$ & $7.86 \pm 0.62$ & $7.90 \pm 0.50$ & $8.10 \pm 0.59$ & $7.53 \pm 0.38$ & $8.17 \pm 0.54$ \\
\hline \multicolumn{9}{|l|}{ Age at MenC, years } \\
\hline Mean \pm SD & $2.79 \pm 0.19$ & $3.56 \pm 0.28$ & $4.56 \pm 0.31$ & $5.49 \pm 0.26$ & $6.41 \pm 0.26$ & $7.46 \pm 0.33$ & $8.44 \pm 0.27$ & $5.07 \pm 1.42$ \\
\hline Range & $(2.38-2.98)$ & (3.05-3.99) & (4.00-4.99) & $(5.02-5.94)$ & $(6.00-6.98)$ & (7.01-7.98) & (8.13-8.79) & $(2.38-8.79)$ \\
\hline
\end{tabular}


Table II: Proportion of participants with serogroup C meningococcal (MenC) serum bactericidal antibody (SBA) titers $\geq 1: 8$, geometric mean MenC serum bactericidal antibody (SBA) titers and MenA, C, W and Y Immunoglobulin G (IgG) antibody geometric mean concentrations 7 to 8 years following MenC vaccination, by age at receipt of primary MenC vaccine

\begin{tabular}{|c|c|c|c|c|c|c|c|c|}
\hline Age at MenC vaccine & 2 years & 3 years & 4 years & 5 years & 6 years & 7 years & 8 years & $\begin{array}{c}\text { All } \\
2 \text { to } 8 \text { years }\end{array}$ \\
\hline $\mathbf{N}$ & 9 & 58 & 67 & 33 & 50 & 16 & 7 & 240 \\
\hline \multicolumn{9}{|l|}{ MenC $r S B A \geq 1: 8$} \\
\hline Proportion & $2 / 9$ & $15 / 58$ & $27 / 67$ & $14 / 33$ & $31 / 49$ & $12 / 16$ & $4 / 7$ & $105 / 240$ \\
\hline$\%$ & 22.2 & 25.9 & 40.3 & 42.4 & 62.0 & 75.0 & 57.1 & 43.8 \\
\hline (95\% C.I.) & $(-11.7-56.1)$ & $(14.3-37.5)$ & $(28.2-52.4)$ & $(24.6-60.2)$ & (48.1-75.9) & $(51.2-98.8)$ & $(7.7-100)$ & $(37.4-50.1)$ \\
\hline \multicolumn{9}{|l|}{ MenC rSBA } \\
\hline GMT & 3.70 & 4.96 & 9.94 & 12.97 & 25.28 & 33.42 & 21.53 & 11.31 \\
\hline (95\% C.I.) & $(1.5-9.1)$ & $(3.2-7.7)$ & $(5.8-16.9)$ & $(5.8-29.1)$ & $(13.8-46.4)$ & $(12.0-92.9)$ & $(2.95-157.3)$ & $(8.67-14.77)$ \\
\hline \multicolumn{9}{|l|}{ MenA GMC } \\
\hline$\mu \mathrm{g} / \mathrm{mL}$ & 1.20 & 1.33 & 1.31 & 1.08 & 1.24 & 1.42 & 1.03 & 1.26 \\
\hline (95\% C.I.) & $(0.59-2.46)$ & $(1.05-1.68)$ & $(1.05-1.63)$ & $(0.77-1.51)$ & $(0.96-1.62)$ & $(0.96-2.08)$ & $(0.57-1.86)$ & $(1.13-1.41)$ \\
\hline \multicolumn{9}{|l|}{ MenC GMC } \\
\hline$\mu \mathrm{g} / \mathrm{mL}$ & 0.13 & 0.23 & 0.27 & 0.38 & 0.36 & 0.30 & 0.52 & 0.29 \\
\hline (95\% C.I.) & $(0.07-0.25)$ & $(0.17-0.31)$ & $(0.21-0.35)$ & $(0.28-0.52)$ & $(0.27-0.50)$ & $(0.15-0.58)$ & $(0.12-2.24)$ & $(0.25-0.33)$ \\
\hline \multicolumn{9}{|l|}{ MenW GMC } \\
\hline$\mu \mathrm{g} / \mathrm{mL}$ & 0.45 & 0.37 & 0.38 & 0.30 & 0.37 & 0.74 & 0.33 & 0.38 \\
\hline (95\% C.I.) & $(0.28-0.72)$ & $(0.30-0.46)$ & $(0.30-0.48)$ & $(0.23-0.40)$ & $(0.29-0.48)$ & (0.36-1.59) & $(0.21-0.52)$ & $(0.34-0.43)$ \\
\hline \multicolumn{9}{|l|}{ MenY GMC } \\
\hline$\mu \mathrm{g} / \mathrm{mL}$ & 0.22 & 0.42 & 0.49 & 0.41 & 0.48 & 0.91 & 0.52 & 0.47 \\
\hline (95\% C.I.) & $(0.11-0.43)$ & $(0.32-0.55)$ & $(0.38-0.64)$ & $(0.26-0.66)$ & $(0.34-0.69)$ & $(0.43-1.91)$ & $(0.27-0.99)$ & $(0.40-0.54)$ \\
\hline
\end{tabular}

GMC: geometric mean concentration, GMT: geometric mean titer, MenA: meningococcal serogroup A, MenC: meningococcal serogroup C, MenW-135: meningococcal serogroup W-135, MenY: meningococcal serogroup Y 
Table III: Proportion of participants with serogroup C meningococcal (MenC) serum bactericidal antibody (SBA) titers $\geq 1: 8$, geometric mean MenC serum bactericidal antibody (SBA) titers and MenC Immunoglobulin G (IgG) antibody concentrations 7 to 8 years following MenC vaccination, by brand of MenC vaccine received

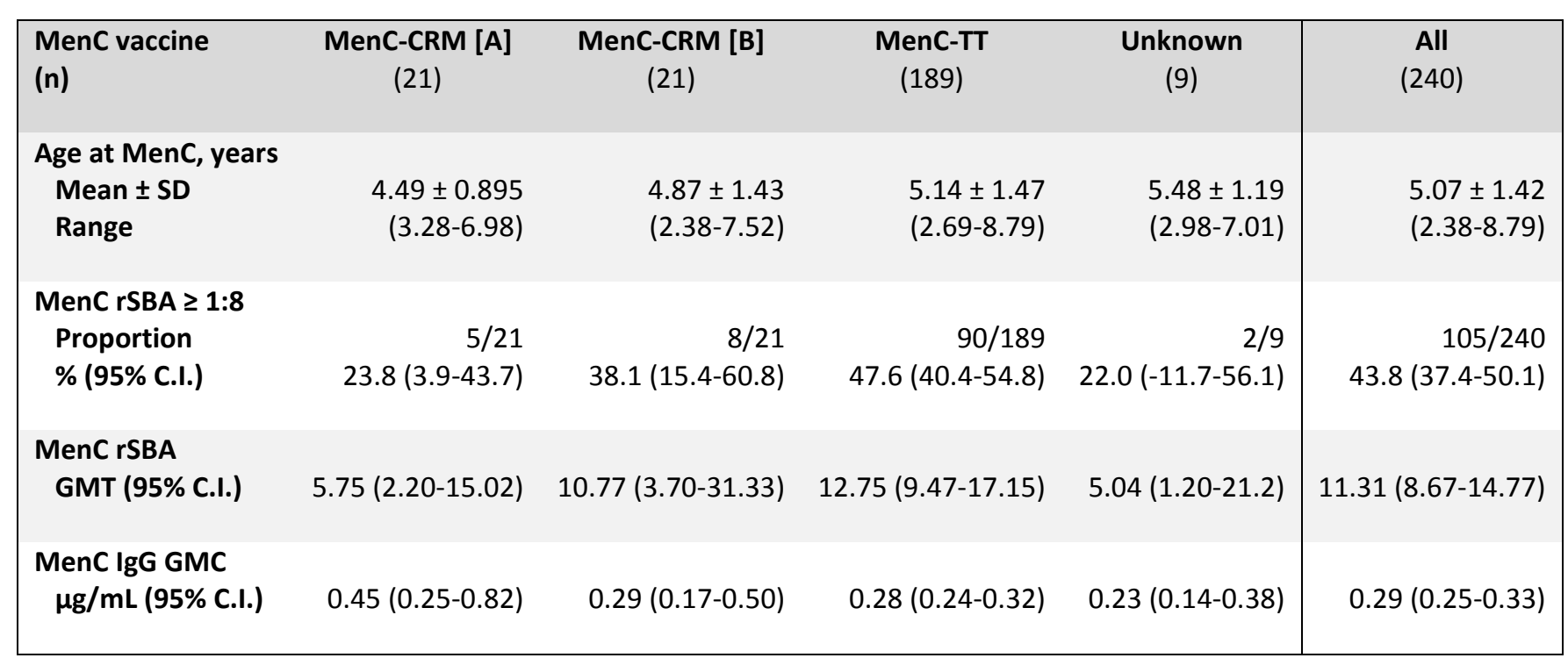

GMC: geometric mean concentration, GMT: geometric mean titer, MenC: meningococcal serogroup C

MenC-CRM [A] = Menjugate, CSL/Novartis Vaccines and Diagnostics

MenC-CRM [B] = Meningitec, Pfizer Australia

MenC-TT = NeisVac-C, Baxter Healthcare 
Table IVa: Linear regression analysis for serogroup C meningococcal (MenC) serum bactericidal antibody (SBA) titers, using rabbit or human complement (rSBA and hSBA), and IgG concentrations, 7 to 8 years following MenC vaccination

\begin{tabular}{|c|c|c|c|c|c|c|c|}
\hline & \multirow[b]{2}{*}{ Parameter } & \multicolumn{3}{|c|}{ Univariate } & \multicolumn{3}{|c|}{ Multivariate } \\
\hline & & Difference & 95\% C.I. & $P$ value & Difference & 95\% C.I. & $\begin{array}{c}P \\
\text { value }\end{array}$ \\
\hline \multirow{3}{*}{$\begin{array}{l}\text { MenC rSBA } \\
\text { titer }\end{array}$} & Age at MenC, years & 0.45 & $0.27,0.63$ & 0.000 & 0.46 & $0.28,0.65$ & 0.000 \\
\hline & Sex, male & 0.08 & $-0.47,0.62$ & 0.780 & -0.08 & $0.62,0.46$ & 0.768 \\
\hline & Brand of MenC* & -0.21 & $-0.65,0.24$ & 0.357 & -0.10 & $-0.53,0.33$ & 0.653 \\
\hline \multirow[t]{3}{*}{ MenC IgG } & Age at MenC, years & 0.15 & $0.05,0.25$ & 0.003 & 0.16 & $0.06,0.26$ & 0.001 \\
\hline & Sex, male & -0.16 & $-0.44,0.13$ & 0.276 & -0.22 & $-0.52,0.07$ & 0.132 \\
\hline & Brand of MenC* & 0.11 & $-0.13,0.34$ & 0.378 & 0.16 & $-0.07,0.39$ & 0.182 \\
\hline \multirow{3}{*}{$\begin{array}{l}\text { ^MenC hSBA } \\
\text { titer }\end{array}$} & Age at MenC, years & 0.10 & $-0.04,0.24$ & 0.155 & 0.10 & $-0.04,0.24$ & 0.163 \\
\hline & Sex, male & -0.33 & $-0.72,0.06$ & 0.097 & -0.31 & $-0.70,0.09$ & 0.124 \\
\hline & Brand of MenC* & -0.34 & $-0.61,0.07$ & 0.014 & -0.29 & $-0.56,-0.02$ & 0.038 \\
\hline
\end{tabular}

Age at MenC, years parameter, refers to each additional year of age at MenC vaccination

* NeisVac-C compared to all CRM conjugates (Menjugate and Meningitec)

^ hSBA GMT were measured in $60 \%$ of participants (140 of the 240 participants)

Table IVb: Logistic regression analysis for the proportion of participants with serogroup $C$ meningococcal (MenC) serum bactericidal antibody (SBA) titers $\geq 8$ (rabbit complement, rSBA) or $\geq 4$ (human complement, hSBA), 7 to 8 years following MenC vaccination

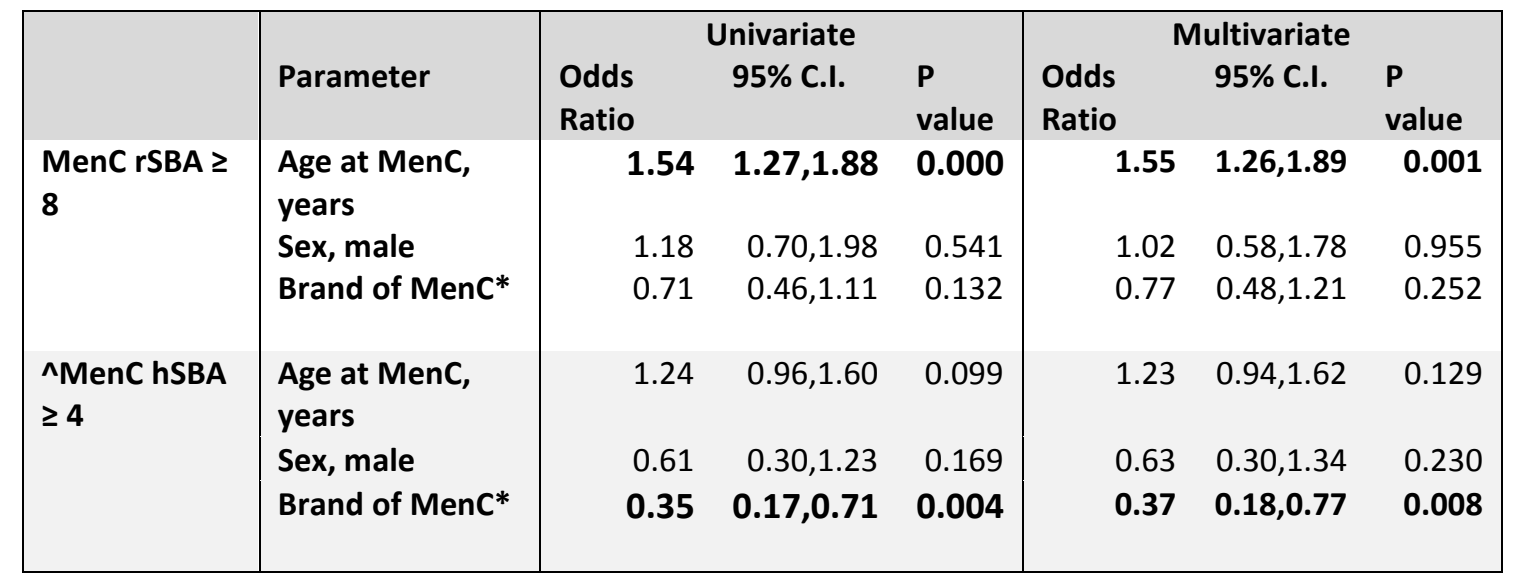

* NeisVac-C compared to all CRM conjugates (Menjugate and Meningitec)

$\wedge$ hSBA GMT were measured in $60 \%$ of participants (140 of the 240 participants) 
Figure 1: Proportion with $\mathrm{rSBA} \geq 8(95 \% \mathrm{CI})$, more than 7 years following MenC vaccine, shown by age at primary MenC vaccination

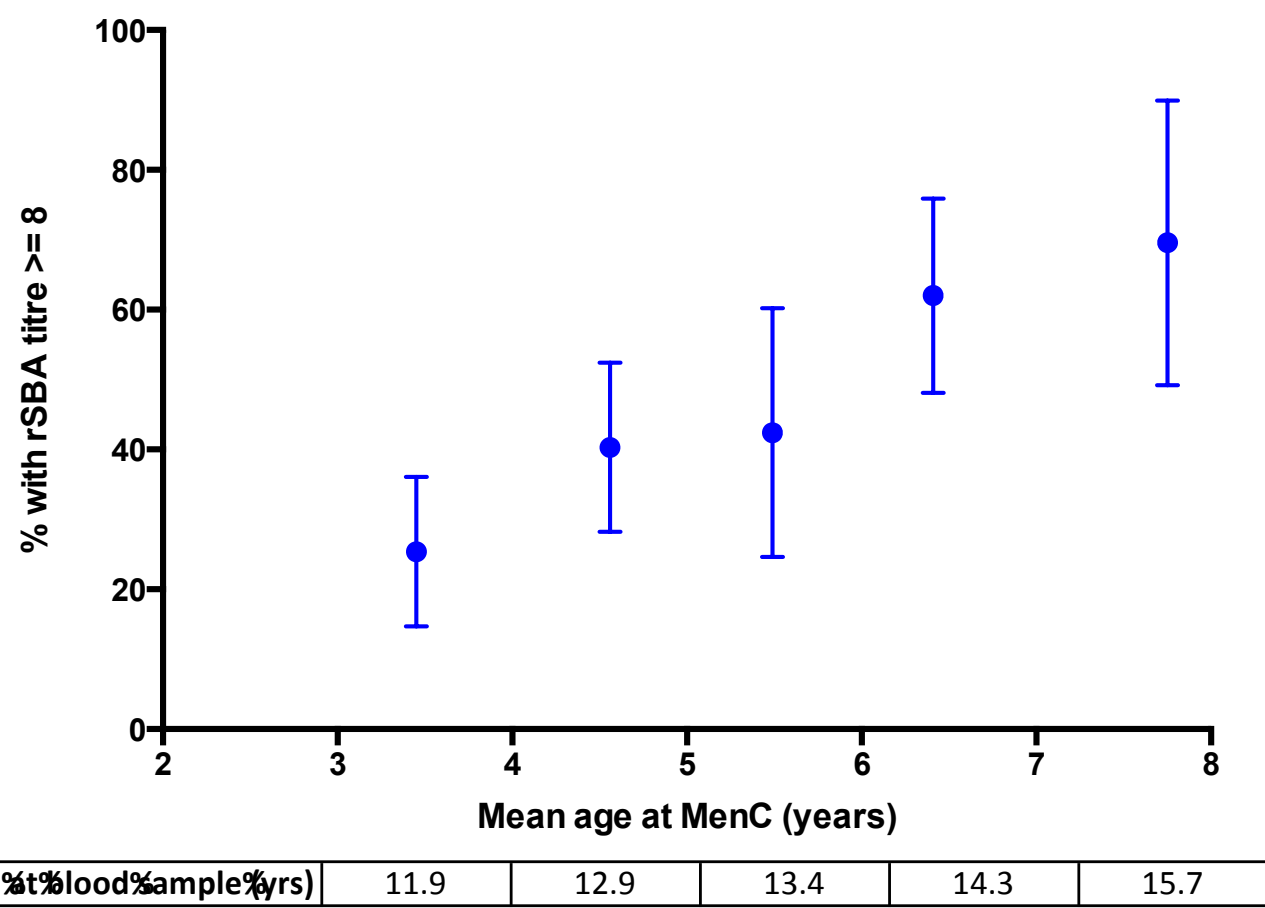




\section{Supplementary Material}

Supplementary Figure 1: MenC SBA geometric mean titers (95\% CIs) 7 to 8 years following MenC vaccine, shown by age at primary MenC vaccination

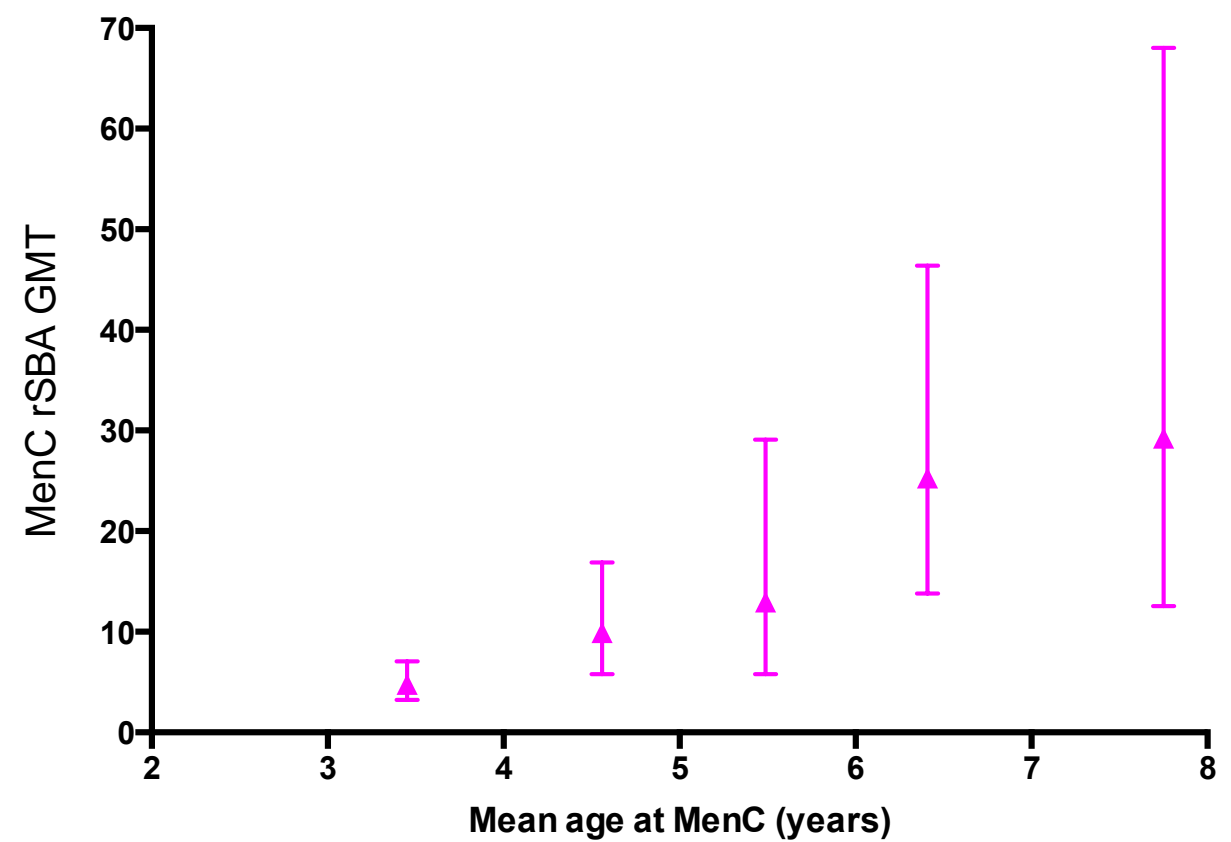

\begin{tabular}{|l|l|l|l|l|l|}
\hline Mean\%ge\%t\%lood\%ample\%yrs) & 11.9 & 12.9 & 13.4 & 14.3 & 15.7 \\
\hline
\end{tabular}

